\title{
Pharmacovigilance and Ethical Issues
}

\author{
Barna Ganguly
}

Professor \& Head, Department of Pharmacology and Head, UNESCO Bioethics Unit, Pramukhswami Medical College, Karamsad, Gujarat, India

Corresponding Author: Barna Ganguly

E-mail - barnaganguly@rediffmail.com

\begin{abstract}
The basic principles of bioethics are beneficence, non-maleficience, autonomy and justice. Pharmacovigilance is explained as a discipline that employs scientific methodologies to detect, assess and understand adverse events associated with use of drugs/biologicals/vaccines. Specific principles of ethics as relevant to Pharmacovigilance are beneficience, nonmaleficience and justice. These are applicable both in regular practice as well as in research especially clinical trial. In the context of developing countries, specifically in India, obtaining informed consent has been considered as a primary requirement which addresses ethical issues like autonomy and justice. In spite of obtaining informed individual consent, it is quite likely that the participants/ patients may not be fully aware of their rights especially in clinical trials in context to serious adverse event (SAE). Ethical guidelines advise that in research involving children and youth, the consent for participation is obtained from their parents or legal guardians. A balance between legality and self autonomy needs to be deliberated upon. Moreover, Investigator's publication plans should not threaten the privacy or confidentiality of participants in whom adverse effects have been detected.
\end{abstract}

Key Words: pharmacovigilance, beneficience, non-malificience, clinical trials, ethics, consent, autonomy, justice.

\section{INTRODUCTION}

The Thalidomide disaster along with other birth defects is known to all of us. About 12,000 babies were born with severe deformities as a result of use of thalidomide as prevention for morning sickness. This was first reported in Lancet [1]. Thalidomide was never approved in the US for morning sickness. But is it only thalidomide? Prior to 1906, when the Pure Food and Drug Act was passed, there were no regulations regarding the ethical use of human participants in research. U.S. Senate hearings followed and in 1962 the so-called "Kefauver Amendments" to the Food, Drug and Cosmetic Act were passed into law to ensure drug efficacy and greater drug safety. For the first time, drug manufacturers were required to prove to the FDA the effectiveness of their products before marketing them [2]. How about other drugs and their safety? In modern medicine how many drugs are there which are free from adverse effects. Adverse Drug Reactions (ADRs) are the 4th to 6th leading cause of death in the USA [3]. In one of the U.K studies, reported that the percentage of hospital admissions due to drug related events in some countries is about or more than $10 \%$ [4]. Another study, it was noted that $16.2 \%$ of hospital admissions are drug-related, in which $54.8 \%$ was due to therapeutic failure, $32.9 \%$ was adverse reactions, $12.3 \%$ of overdosing. Out of all, $49.3 \%$ could have been avoided [5]. 


\section{What is Pharmacovigilance?}

WHO has defined it as the science and activities relating to the detection, evaluation, understanding and prevention of adverse drug reactions or any other drug-related problem. This has also been explained as a discipline that employs scientific methodologies to detect, assess and understand adverse events associated with use of drugs/biologicals/vaccines [6].

\section{Situation in India}

In India, more and more new drugs are being introduced which include New Chemical Entities (NCE), high tech pharma products, vaccines as well as new dosage forms, new routes of drug administrations and new therapeutic claims of existing drugs. Such a rapid inflow of NCEs and high tech Pharma products in the market throw up the challenges of monitoring Adverse Drug Reactions (ADRs) over large population base. All medicines (pharmaceuticals and vaccines) have side effects. Some are known, while many are still unknown even though lot of medicines has been in clinical use for several years. In a vast country like India with a population of over 1.2 billion with vast ethnic variability, different disease prevalence patterns, practice of different systems of medicines, different socioeconomic status, it is important to have a standardized and robust pharmacovigilance and drug safety monitoring programme for the nation. Collecting this information in a systematic manner and analyzing the data to reach a meaningful conclusion on the continued use of these medicines is the rationale to institute Pharmacovigilance program of India by CDSCO. Since, there are considerable social and economic consequences of ADRs there is a need to engage health-care professionals, in a well structured programme to build synergies for monitoring ADRs. But the origin of pharmacovigilance in India goes back to 1986, when a formal adverse drug reaction (ADR) monitoring system consisted of 12 regional centers, each covering a population of 50 million was established. Unfortunately, the then collected data was not published so we can consider that the outcome of the entire program was a failure. India is a hub for clinical trials flooded with more than 6,000 licensed drug manufacturers and 60,000 branded formulations. Pharmacovigilance is an important part of clinical research and practice, yet there is an immense need to understand the importance of pharmacovigilance. Thus the Pharmacovigilance Programme of India (PvPI) was launched in 2012 with an objective to safe guard the health of people of India. While major advancements in this discipline have taken place in West, implementation and compliance still remain as challenge in India. In India, the events are not properly reported due to lack of time, low motivation, ignorance. Lack of continuing medical education on pharmacovigilance and dearth of drug information particularly at the level of primary health centres and private practitioners lead to under-reporting of ADR. The practice of self-medication, use of traditional medicines, etc pose other challenges as adverse events in such cases often go unreported.

\section{Aim of Bioethics}

The basic principles of bioethics are beneficence, non-maleficience, autonomy and justice. While keeping general ethical principles of respect for persons in mind, duty to maximize possible benefits and minimize possible harm are important considerations in ethical guidelines. All ethical concerns and issues are to be seen in the socio-cultural context to which they are intended to be used in the country. The issues and perceptions should be dynamic and not static, in order to address changing requirements and future challenges.

Specific principles of ethics as relevant to Pharmacovigilance are beneficience, non-maleficience and justice. These are applicable both in regular practice as well as in research especially clinical trials.

\section{Clinical trials}

A clinical trial is a systematic study involving human participants intended to generate data to find out the safety and efficacy of medications, devices, diagnostic products, herbal or herbomineral remedies and preventive measures like vaccines etc. There are certain risks for the participants in such trials, which involve healthy volunteers and patients [7]. There have been a set of guidelines that have been laid down to conduct the evaluation of research interventions in 
human beings and to protect the research participants from any avoidable risks, to guide researchers while planning trial protocols in specific areas such as -

1. Drug Trials.

2. Vaccine Trials.

3. Clinical Trials with Surgical Procedures or Medical Devices.

4. Trials with Diagnostic agents-with special reference to use of Radio Active Materials and X-Rays.

5. Trials with Herbal Remedies.

6. Trials with emerging therapies such as gene therapy, biologicals, stem cell therapy etc. The safety of the trial subjects is the sole responsibility of the investigator. The investigator should be medically qualified and should be compliant with the protocol. S/he should conduct the trial strictly abiding by Indian GCP guidelines and ICH-GCP Guidelines if the requirement is by USFDA or EU regulatory agencies. The investigator should ensure the protection of the rights, safety and well-being of the trial participants.

While conducting clinical trials, the concerned study personnel should stick to the four basic ethical principles which are-

1. Autonomy or Respect for the Study Participants.

2. Beneficence or Do good.

3. Non-Maleficence or Do no harm.

4. Justice.

When individuals are to be included as participants of drug trials in new drug development, the purpose and general objectives of the study has to be explained to them keeping in mind their level of understanding. In the context of developing countries, specifically in India, obtaining informed consent has been considered as a primary requirement, which addresses ethical issues like autonomy and justice. In spite of obtaining informed individual consent, it is quite likely that the participants/ patients may not be fully aware of their rights. To ensure this, CDSCO has introduced guidelines to follow, such as audiovisual recording of informed consent process to ensure that the participants are well aware of the process. The role of investigator is crucial and $\mathrm{s} /$ he should remain vigilant and conscious of her/ his obligations towards the participants/ patients, all through the course of the studies. The issue of compensation for accidental injury has been taken up by the Indian Regulators and has also been finalized.

\section{Other research}

Different kinds of research (epidemiological studies, post marketing surveillance, other pharmacovigilance studies, clinical trials, product development) have their own particular scientific requirements and specific ethical challenges. Hence the choice of study populations for each type of study should be justified in advance in scientific and ethical terms. This choice should be regardless of the place from where the study population is selected. Generally, early clinical phases of research, particularly of drugs, vaccines and devices, should be conducted in communities that are less vulnerable to harm or exploitation. However, for valid scientific and public health reasons, if sufficient scientific and ethical safeguards are ensured, the research may be conducted in any phase after obtaining relevant regulatory clearances.

Ethical guidelines advise that in research involving children and youth, the consent for participation is obtained from their parents or legal guardians. A balance between legality and self autonomy needs to be deliberated upon.

Finally, Principal Investigator's publication plans should not threaten the privacy or confidentiality of participants. The ICMR ethical guidelines recommend that a clear consent for publication be obtained along with the consent for participation in research or treatment. Such consent should preferably be obtained on two different occasions and not as a blanket one at the commencement of the study.

\section{Regular practice}

Adverse drug reactions (ADRs) are one of the major causes of hospital admission and in-hospital morbidity. They have become an important clinical problem and a constant concern of the public 
healthcare systems accounting for up to $5 \%$ of hospital admission, $28 \%$ of emergency visits and $5 \%$ of hospital deaths with associated costs of economic burden. Therefore, timely and accurate detection of adverse reactions is critical in improving patient's safety and therefore reducing healthcare costs. This may be considered one of the ethical principles in line with beneficence and justice for the patients.

Drug safety surveillance has relied predominantly on spontaneous reporting system, which involves voluntary reporting of suspected ADRs by healthcare professionals including clinicians, nurses and pharmacists. It is obvious that involvement of paramedical staff in spontaneous reporting of $\mathrm{ADR}$ is essential and helps in improving the reporting quality and rates. Hospital nurses may play an important role in ADRs reporting, because they are in close contact with the patients for a longer duration and have good knowledge of health management, symptoms, drugs and ADRs. In such a situation, autonomy of the patients may be compromised but safety and benefits are definitely being taken care of. Similarly, patients have a right to privacy and also right to get information about any condition or disease from which they are suffering. The principle of confidentiality that acknowledges respect for a patient's privacy, is the fundamental obligation to be observed by the entire healthcare system. As the ability to quickly give and receive information has increased, concerns about the privacy and confidentiality of personal information have also greatly increased. This also highlights the fine balance between patients' rights, public safety and medical progress.

Recently Public Health Ethics is an emerging discipline which focuses on addressing ethical issues related to research or interventions that are to be considered not only at individual level but also concerning the health of population at large or in groups [8].

As Public Health encompasses surveillance, interventions, screening etc. the implication of ethical issues related to pharmacovigilance in line with Public Health Ethics. This may lead to a new perspective and some new arguments which can be solved or simplified through moral reasoning with the help of judgment and rational analysis.

\section{REFERENCES}

1. Dally A. Thalidomide: was the tragedy preventable? Lancet 1998;351(9110):1197-9.

2. History of Ethics - Claremount Graduate University. www.cgu.edu/pages/1722.asp ( accessed on 30.12.2015)

3. Lazarou J, Pomeranz BH, Corey PN. Incidence of adverse drug reactions in hospitalized patients: a meta analysis of prospective studies. JAMA 1998;279:1200-5

4. Bhalla N, Duggan C, Dhillon S. The incidence and nature of drug-related admissions to the hospital. Pharm. J 2003;270:583-6.

5. Nelson KM, Talbert RM. Drug-related hospital admissions. Pharmacotherapy 1996;16:701-7.

6. World Health Organization (WHO). The importance of pharmacovigilance: safety monitoring of medicinal products (online). http://apps.who.int/medicinedocs/pdf/s4893e/s4893e.pdf. Accessed 25 Dec 2015.

7. Indian Council of Medical Research (ICMR). Statement of the general principles in biomedical research involving human participants. Ethical Guidelines for Biomedical Research on Human Participants, 2006: Chapter III, 21-33.

8. Callreus T. Pharmacovigilance and Public Health Ethics. Curr Opin Pharmaceut Med 2013;27(3):157-64.

Acknowledgements - Nil

Source of Funding - Nil

Conflict of Interest - Nil 